\title{
Le Principe de Jordan et la paralysie des administrations publiques
}

$\mathrm{L}$ es enfants sont des membres vulnérables de notre société. Ils n'ont pas voix au chapitre pour la prise de décisions, ils sont soumis aux jugements et aux actions d'autrui. Les gens des Premières nations aussi sont vulnérables - victimes de la mauvaise volonté et des promesses rompues, ils souffrent des pires conditions sociales, économiques et de santé au Canada.

Imaginez alors la situation peu enviable d'un enfant très malade des Premières nations qui habite dans une réserve presque démunie de services aux enfants.

À sa naissance, en 1999, dans la réserve de la nation Cri de Norway House, au nord du Manitoba, "Jordan» était atteint d'un trouble neuromusculaire rare ${ }^{1}$. Il était impossible de répondre à ses besoins médicaux complexes chez lui et il a été envoyé à Winnipeg pour traitement. La maladie s'est aggravée, il a été confiné au fauteuil roulant et mis sous ventilateur, et il ne pouvait parler.

Les soignants de l'hôpital ont décidé en 200I de confier Jordan à une famille d'accueil spécialisée vivant près de sa réserve natale. Les médecins et la famille ont convenu que c'était la meilleure décision pour Jordan. Les bureaucrates ont ensuite tout gâché. Le gouvernement fédéral et le gouvernement du Manitoba n'ont pu décider d'un commun accord qui serait financièrement responsable des soins de Jordan. Le désaccord portait sur le choix de la famille d'accueil, sur le transport vers la clinique et même sur de menus détails comme une pomme de douche. Les bureaucrates belliqueux ont tout examiné à la loupe pendant plus de deux ans.

Cette dispute intergouvernementale a pris fin seulement, vous l'avez deviné, lorsque Jordan est décédé de sa maladie première dans un hôpital de Winnipeg, loin de sa famille et de sa communauté. Personne n'a été tenu responsable d'avoir empêché Jordan d'obtenir des soins plus près de son domicile.

Le Canada a ratifié en 1999 la Convention des Nations Unies sur les droits de l'enfant, qui énonce ce qui suit : «Dans toutes les décisions qui concernent les enfants, qu'elles soient le fait des institutions publiques ou privées de protection sociale, des tribunaux, des autorités administratives ou des organes législatifs, l'intérêt supérieur de l'enfant doit être une considération primordiale»².

Les intérêts de Jordan sont passés loin derrière les désaccords intergouvernementaux sur le devoir de payer. Le Canada a ainsi enfreint la Convention.

La Charte des droits et libertés du Canada interdit la discrimination. Nombre des services dont Jordan avaient besoin auraient été payés sans question pour un résident de race blanche du Manitoba ou un résident autochtone hors réserve. Or, Jordan habitait dans une réserve, ce que n'a pu avaler la bureaucratie. Voilà de la discrimination, pure et simple.

La Constitution du Canada reconnait et affirme les droits des traités et des Autochtones. La Cour suprême a déclaré en I984: «(...) le gouvernement a la responsabilité d'agir en qualité de fiduciaire à l'égard des peuples autochtones» dans une relation «(...) de nature fiduciaire plutôt que contradictoire». On se demande comment les bureaucrates ont respecté cette obligation lorsqu'ils ont laissé Jordan vivre et mourir ensuite à l'hôpital de Winnipeg loin de ses proches pendant que cette guerre intestine faisait rage.

D'autres enfants des Premières nations qui ont des besoins médicaux complexes sont souvent pris au piège de cauchemars bureaucratiques semblables. En mars dernier, on a dit aux familles de 37 enfants profondément handicapés de la nation Cri de Norway House que les services professionnels de santé et les services de soutien ne seraient plus financés dans leur communauté ${ }^{3}$. Les familles qui voulaient des soins de santé pour leurs enfants ont dû les envoyer au loin, sans doute pour toujours.

Ceux qui défendent le statu quo affirment que la prestation des soins de santé pour les maladies chroniques complexes est difficile et coûteuse à cause de la géographie du Canada. Les mêmes critiques omettent habituellement de mentionner que la géographie enrichit aussi le Canada avec le pétrole, le bois d'œuvre, les minéraux et les voies navigables en majeure partie sur le territoire traditionnel des Premières nations. La géographie n'excuse pas une répartition inéquitable et pusillanime de la richesse en raison de laquelle les soins de pointe sont disponibles seulement au sud tandis que des enfants, parents et communautés des Premières nations doivent endurer un stress psychologique et culturel pour y avoir accès. Il ne s'agit pas de déterminer quel pourcentage des coûts les gouvernements provinciaux, territoriaux et fédéral doivent payer, mais plutôt de mettre fin aux tergiversations pour simplement dispenser les soins appropriés, au bon endroit et au bon moment, aux habitants des réserves des Premières nations.

Le JAMC adopte aujourd'hui le «Principe de Jordan» (www.fncfcs .com/more/jordansPrinciple_f.php). Conformément à la Convention relative aux droits de l'enfant, nous affirmons que les besoins médicaux des enfants des Premières nations doivent passer en premier. Nous ajoutons la recommandation suivante : si les gouvernements fédéral, provinciaux et territoriaux ignorent le Principe de Jordan et s'enferrent au départ dans des batailles financières ou de compétence, ils méritent alors d'être traduits en justice dans cette affaire de principe la plus susceptible d'être gagnée dont puissent se charger les défenseurs des Premières nations. Si les bureaucrates et les politiciens refusent toujours de trouver rapidement une solution, que les tribunaux tranchent.

\section{Noni MacDonald MD MSc}

Rédactrice de la section Santé publique, JAMC

Amir Attaran LLB PhD

Titulaire de la Chaire de recherche du Canada en Santé de la population et politique du développement mondial Université d'Ottawa, Ottawa, Ont.

Pour l'équipe de rédaction de l'éditorial (Paul C. Hébert, Matthew Stanbrook, Barbara Sibbald and Ken Flegel)

Remerciements : Nous remercions les personnes suivantes de leur contrubution à cet éditorial : Cindy Blackstock, MM, directrice générale, Société de soutien à l'enfance et à la famille des Premières Nations du Canada, Ottawa; Bradford W. Morse, LLM, professeur de droit, Université d'Ottawa; Jeff Reading, PhD PHS, directeur scientifique, Institut de la santé des Autochtones des IRSC, Université de Victoria, Victoria.

\section{RÉFÉRENCES}

I. Lavallée, T.L. Honouring Jordan: Putting First Nations children first and funding fights second. J Paediatr Child Health 2005;10:527-9.

2. Convention des Nations Unies sur les droits de l'enfant. Disponible à : www.ohchr .org/french/law/crc.htm (consulté le 9 juillet 2007).

3. Assemblée des chefs du Manitoba. Disabled children lose services because governments won't pay. Disponible à : www.manitobachiefs.com/press/norway-house .pdf (consulté le is juin 2007). 